\title{
Salvage Robot-Assisted Laparoscopic Prostatectomy: Tips and Tricks for Challenges Caused by Different Primary Treatments
}

\section{Marcio Covas Moschovas, MD}

Department of Urology, AdventHealth Global Robotics Institute, Florida Hospital, Celebration, Florida. E-mail: marcio.doc@hotmail.com

Fikret F. Önol, MD

Department of Urology, AdventHealth Global Robotics Institute, Florida Hospital, Celebration, Florida.

Seetharam Bhat, MD

Department of Urology, AdventHealth Global Robotics Institute, Florida Hospital, Celebration, Florida.

\section{Travis Rogers, MD}

Department of Urology, AdventHealth Global Robotics Institute, Florida Hospital, Celebration, Florida.

\section{Shannon Roof, MD}

Department of Urology, AdventHealth Global Robotics Institute, Florida Hospital, Celebration, Florida.

Vipul Patel, MD

Department of Urology, AdventHealth Global Robotics Institute, Florida Hospital, Celebration, Florida.

(c) Mary Ann Liebert, Inc. DOI: 10.1089/vid.2019.0045
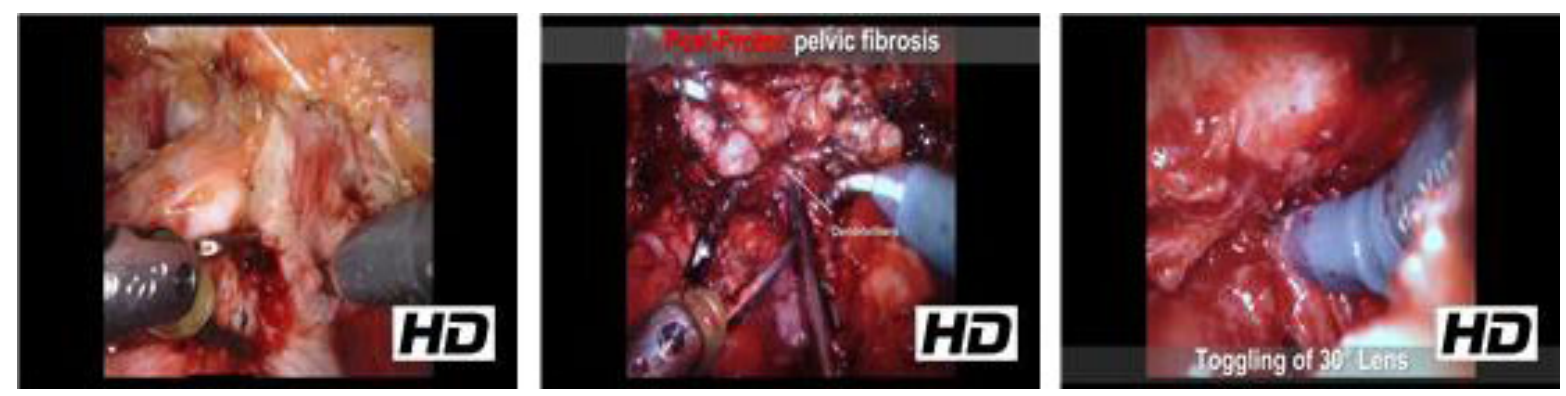

\section{Abstract}

Introduction and Objectives: Salvage robot-assisted laparoscopic prostatectomy (sRALP) is a technically demanding procedure because of significant alterations in anatomical landmarks and loss of tissue planes. In this video, we present the challenges associated with different primary treatments and describe key points in their management.

Materials and Methods: Between 2008 and 2018, 126 patients underwent SRALP by a single experienced surgeon. All procedures were performed using a transperitoneal six-port technique. Ninety-four patients had received external or internal radiation (EBRT: 39, IMRT: 15, proton beam: 3 , brachytherapy: 23, EBRT+brachy: 14) and 32 had received focal therapy (cryotherapy: 20, HIFU: 9 , microwave/electroporation: 3 ) as primary treatment. Difficulties caused by different primary therapies at each surgical step were identified and key points in their management were presented.

Results: (1) Endopelvic fascia dissection (EFD): ipsilateral pelvic side-wall fibrosis and troublesome bleeding from vessels obscured by scarring were common after cryoablation. Opening the endopelvic fascia at the prostate base where the space between the prostate and the levators is least vascular and dissection away from the prostate capsule toward the apex facilitated EFD. Apical dissection around the sphincter was typically difficult in brachytherapy patients because of inflammation caused 
by misplaced seeds. (2) Bladder neck dissection (BND): in cases with HIFU, the prostate was atrophic and fused anteriorly behind the pubic tubercle. In such cases, opening the anterior BN provided better identification of the anatomy followed by antegrade prostatectomy. (3) Posterior dissection (PD): IMRT and proton beam were noted to cause more extensive fibrosis in the pelvis. In such cases, identification of the correct plane for PD was especially difficult because of lack of prerectal fat and "tenting" of the rectum. We have used the instant toggling feature of DaVinci Xi robot $\left(180^{\circ}\right.$ upward rotation of the $30^{\circ}$ camera) to facilitate observation of the posterior plane and careful cold dissection. (4) Vesicourethral anastomosis (VUA): loss of tissue vascularization after EBRT and proton beam radiation adversely affects VUA vitality and leads to anastomotic dehiscence or leaks. In our experience, use of an acellular and resorbable scaffold graft to reinforce base of the VUA resulted in decreased leak rates and catheterization times. ${ }^{1}$

Conclusions: SRALP should be performed by experienced surgeons because of lack of tissue planes and anatomical landmarks. The surgeon should be familiar with challenges specific to different primary therapies. Opening endopelvic fascia away from prostate capsule, antegrade prostatectomy when prostate and $\mathrm{BN}$ are fused to the pubis, $30^{\circ}$ toggling during $\mathrm{PD}$, and Acell graft to reinforce VUA are keys in management of complex cases.

No competing financial interests exist.

Runtime of video: 8 mins 46 secs

Keywords: challenging cases, robotic radical prostatectomy, robotic salvage prostatectomy

\section{Cite this video}

Marcio Covas Moschovas, Fikret F. Önol, Seetharam Bhat, Travis Rogers, Shannon Roof, Vipul Patel, Salvage Robot-Assisted Laparoscopic Prostatectomy: Tips and Tricks for Challenges Caused by Different Primary Treatments, VideoEndocrinology. 2019, DOI: 10.1089/vid.2019.0045.

\section{Reference}

1. Ogaya-Pinies G, Palayapalam-Ganapathi H, Rogers $\mathrm{T}$, et al. Can dehydrated human amnion/chorion membrane accelerate the return to potency after a nerve-sparing robotic-assisted radical prostatectomy? Propensity score-matched analysis. J Robotic Surg 2018;12:235-243.

Original Publication Date: 2019 